\title{
Automatisierte Kontrolle von Teile-Oberflächen
}

\author{
Ein Hersteller von Kfz-Präzisionsteilen aus Metall nutzt ein Bildverarbeitungssystem, um die \\ Oberflächenqualität von Bauteilen wie zum Beispiel Überwurfmuttern für Benzinleitungen zu prüfen.
}

A ls Hersteller von Präzisionsteilen aus Kupfer- und Stahllegierungen beliefert die Leipold Gruppe unter anderem die Automobilindustrie mit Komponenten für Kraftstoffführungen, Sensoren und Steuergeräte. Das Stammhaus der Gruppe in Wolfach/ Schwarzwald gehört zu den ältesten industriellen Drehereien in Deutschland. Weitere Werke befinden sich in Bünde, Dransfeld und Windsor/ USA.

\section{Oberflächenqualität wird per Kamera geprüft}

Zu den Kernkompetenzen der LeipoldGruppe gehört die Produktion von Kugelbuchsen und Überwurfmuttern für Hoch- und Niederdruckbenzinleitungen. Diese sicherheitsrelevanten Drehteile müssen mit sehr hoher Präzision gefertigt und zu 100 Prozent geprüft werden. Dabei kommen hoch automatisierte Prüfanlagen zum Einsatz, die speziell für diese Komponenten entwickelt wurden und einen sehr hohen Durchsatz erreichen.

Bisher wurden diese Prüfanlagen hauptsächlich für die geometrische Prüfung der Bauteile eingesetzt. Jetzt betreibt Leipold an zwei Standorten

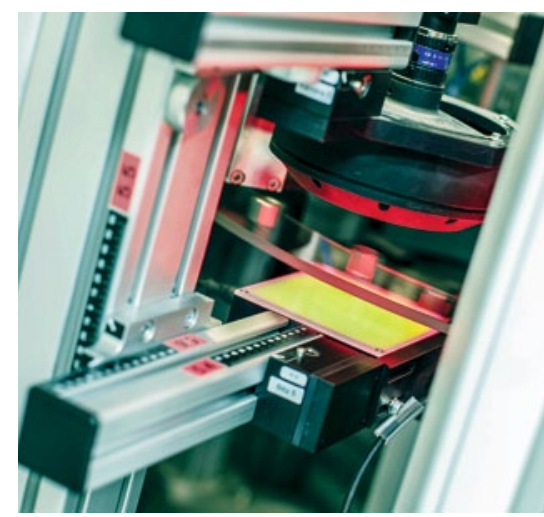

Anlagen, die darüber hinaus auch die Oberflächenqualität der Komponenten inspizieren. Gemeinsam mit dem Anlagenhersteller, der Vester Elektronik GmbH in Straubenhardt, wurden die Kriterien für Gut- und Schlechtteile definiert.

\section{Lohnend bei sehr hohem \\ Durchsatz}

Die Teile werden kontinuierlich der Anlage zugeführt und durchlaufen auf einem rotierenden Glasteller fünf Prüfstationen, in denen sie in einer 360-Grad-Kontrolle aus allen Blickwinkeln aufgenommen werden. Entdeckt die Anlagensteuerung einen Makel, fällt das Präzisionsteil in den Schlecht-Behälter. Als gut erkannte Artikel werden der Weiterverarbeitung zugeführt.

Die Leipold Gruppe setzt die vollautomatische Kameraprüfung bisher ganz bewusst nur bei der Kontrolle zweier Typen von Präzisionsteilen ein. Pascal Schiefer, Vorsitzender der Geschäftsführung: „Weil das Prüfverfahren hoch spezialisiert und auf die Anforderungen eines Teils abgestimmt ist, lohnt es sich nur bei großen Losgrößen."

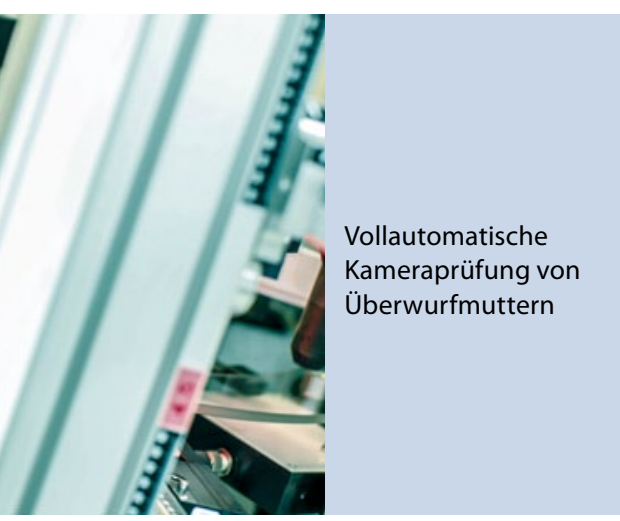

Am Standort Dransfeld im südlichen Niedersachsen inspizieren schon seit einiger Zeit Kameraprüfsysteme die Qualität von Kugelbuchsen. Die Ergebnisse waren rundum überzeugend. Deshalb wurde das Prüfverfahren jetzt auch am Hauptsitz in Wolfach eingeführt und auf Überwurfmuttern angepasst.

\section{Sicherheitssensible}

\section{Präzisionskomponenten}

Kugelbuchsen und Überwurfmuttern kommen an den elementaren Schnittstellen der Kraftstoffzufuhr bei Verbrennungsmotoren teilweise mit Betriebsdrücken von bis zu 200 bar zum Einsatz. Beide Teile-Typen fertigt das Unternehmen jeweils in einer Menge von sechs bis acht Millionen Stück pro Jahr. Eine besondere prüftechnische Herausforderung stellte die Risserkennung an den Sechskantflächen der Überwurfmutter dar. Durch Risse in der Oberfläche, die bereits im Rohmaterial vorkommen und nur selten Folge der spanenden Fertigung sind, könnte Kraftstoff austreten oder das Teil durch die starke Beanspruchung sogar aufbrechen. Diese Fehlerquelle muss daher unbedingt vermieden werden. Die automatisierte Prüfung der Oberflächenbeschaffenheit per Bildverarbeitung stellt sicher, dass fehlerhafte Teile zuverlässig erkannt und direkt aus dem Produktionsprozess ausgeschleust werden. I

\section{Kontakt:}

Carl Leipold GmbH, Wolfach,

Tel. 0783483950 ,

info@leipold.com,

www.leipold.com 